\title{
Asynchronous Convolutional-Coded Physical-Layer Network Coding
}

\author{
Qing Yang, Student Member, IEEE, and Soung Chang Liew, Fellow, IEEE
}

\begin{abstract}
This paper investigates the decoding process of asynchronous convolutional-coded physical-layer network coding (PNC) systems. Specifically, we put forth a layered decoding framework for convolutionalcoded PNC consisting of three layers: symbol realignment layer, codeword realignment layer, and joint channel-decoding network coding (Jt-CNC) decoding layer. Our framework can deal with phase asynchrony and symbol arrival-time asynchrony between the signals simultaneously transmitted by multiple sources. A salient feature of this framework is that it can handle both fractional and integral symbol offsets; previously proposed PNC decoding algorithms (e.g., XOR-CD and reduced-state Viterbi algorithms) can only deal with fractional symbol offset. Moreover, the Jt-CNC algorithm, based on belief propagation (BP), is BER-optimal for synchronous PNC and near optimal for asynchronous PNC. Extending beyond convolutional codes, we further generalize the Jt-CNC decoding algorithm for all cyclic codes. Our simulation shows that Jt-CNC outperforms the previously proposed XOR-CD algorithm and reduced-state Viterbi algorithm by $2 \mathrm{~dB}$ for synchronous PNC. For phase-asynchronous PNC, Jt-CNC is $4 \mathrm{~dB}$ better than the other two algorithms. Importantly, for real wireless environment testing, we have also implemented our decoding algorithm in a PNC system built on the USRP software radio platform. Our experiment shows that the proposed Jt-CNC decoder works well in practice.
\end{abstract}

\section{Keywords}

Physical-layer network coding; convolutional codes; symbol misalignment; phase offset; joint channeldecoding and network coding.

The authors are with the Department of Information Engineering, The Chinese University of Hong Kong, Shatin, New Territories, Hong Kong (email: \{yq010, soung\}@ie.cuhk.edu.hk).

This work is partially supported by AoE grant E-02/08 and the General Research Funds Project No. 414911, established under the University Grant Committee of the Hong Kong Special Administrative Region, China. This work is also partially supported by the China 973 Program, Project No. 2012CB315904. 


\section{INTRODUCTION}

This paper investigates the use of convolutional codes in asynchronous physical-layer network coding (PNC) systems to ensure reliable communication. In particular, we focus on the decoding problem when simultaneous signals from multiple transmitters arrive at a PNC receiver with asynchronies between them.

PNC was first proposed in [1] as a way to exploit network coding [2], [3] at the physical layer. In the simplest PNC setup, two users exchange information via a relay in a two-way relay network (TWRN). The two users transmit their messages simultaneously to the relay; the relay then maps the overlapped signals to a network-coded message and broadcasts it to the two users; and each of the two users recovers the message from the other user based on the network-coded message and the knowledge of its own message. PNC can potentially boost the throughput of TWRN by $100 \%$ compared with a traditional relay system [1].

Our paper focuses on PNC decoding as applied to TWRN. To ensure reliable transmission, communication systems make use of channel coding to protect the information from noise and fading. In channel-coded PNC, the goal of the relay is to decode the simultaneously received signals not into the individual messages of the two users, but into a network-coded message. This process is referred to as the channel-decoding network coding (CNC) process in [4].

In addition to the issue of channel coding, in practice, the signals from the two users may be asynchronous in that there may be relative symbol arrival-time asynchrony (symbol misalignment), phase asynchrony (phase offset), and other asynchronies between the two signals received at the relay. These PNC systems are referred to as asynchronous PNC (APNC) systems [5].

Both [4] and [5] assume the use of repeat accumulate (RA) codes. Our current paper, on the other hand, focuses on the use of convolutional codes. A main motivation is that convolutional codes are commonly adopted in many communications systems (e.g., the channel code in IEEE 802.11 is a convolutional code [6]). Convolutional codes have been well studied and there are many good designs for the encoding/decoding of convolutional codes in the conventional communication setting. Given this backdrop, whether these designs are still applicable to PNC, and what additional considerations and modifications are needed for PNC, are issues of utmost interest. This paper is an attempt to address these issues.

Our main contributions are as follows:

- We put forth a layered decoding framework for asynchronous PNC system. The proposed decoding framework can deal with synchronous PNC as well as asynchronous PNC with relative phase offset 
and general symbol misalignment—by general symbol misalignment, we mean that the arrival times of the two users' signals at the relay are offset by $\left(\tau_{\mathrm{I}}+\tau_{\mathrm{F}}\right)$ symbol durations, where $\tau_{\mathrm{I}}$ is an integral offset and $\tau_{\mathrm{F}}$ is a fractional offset smaller than one.

- We design a joint channel-decoding network coding (Jt-CNC) decoder for convolutional-coded PNC. The Jt-CNC decoder, based on belief propagation (BP), is optimal in terms of bit error rate (BER) performance.

- We implement the Jt-CNC decoder in a real PNC system built on USRP software radio platform. Our experiment shows that the Jt-CNC decoder works well under real wireless channel.

- We propose an algorithm that can handle general symbol misalignment in cyclic-coded PNC, building on the insight obtained from our study of convolutional-coded PNC; that is, the algorithm is applicable to all cyclic codes, not just convolutional codes.

The remainder of this paper is organized as follows. Section III overviews related work. Section III describes the PNC system model. Section IV] puts forth our Jt-CNC framework, focusing on synchronous PNC. Section V/extends the Jt-CNC framework to asynchronous PNC. We further show how the algorithmic framework is actually applicable to the general cyclic-coded PNC. Section VI presents simulations and experimental results. Section VII concludes this work.

\section{RELATED WORK}

\section{A. Synchronous PNC with Convolutional Codes}

The first implementation of TWRN based on the principle of PNC was recently reported in [7], [8]. This system employs the convolutional code defined in the 802.11 standard and adopts the OFDM modulation to eliminate symbol misalignment [9]. In [7], [8], first the log-likelihood ratio (LLR) of the XORed channel-coded bits is computed; then this soft information is fed to a conventional Viterbi decoder. We refer to this decoding strategy as the soft XOR and channel decoding (XOR-CD) scheme [10]. The experiment shows that the use of XOR-CD on the convolutional-coded PNC system, thanks to its simplicity, is feasible and practical.

The acronym XOR-CD refers to a two-step process: first, prior to channel decoding and without considering the correlations among the received symbols due to the channel code, we apply symbol-bysymbol PNC mapping on the received symbols to obtain estimates on the successive XORed bits; after that, we perform channel decoding on the XORed bits to obtain the XORed source bits. The performance of XOR-CD is suboptimal because the PNC mapping in the first step loses information [4]. Furthermore, only linear channel codes can be correctly decoded in the second step. Jt-CNC, on the other hand, 
performs channel decoding and network coding as an integrated process rather than two disjoint steps. Jt-CNC can be ML (maximum likelihood) optimal, depending on which variations of Jt-CNC we use and whether the underlying PNC system is synchronous or asynchronous.

Within the class of Jt-CNC algorithms, for optimality, there are two possible decoding targets: (i) ML XORed codeword; (ii) ML XORed bits. To draw an analogy, for the conventional single-user point-topoint communication, if convolutional codes are used, then the Viterbi algorithm [11] aims to obtain the ML codeword, while the BCJR [12] aims to obtain ML bits. For PNC systems, the aim is to obtain the network-coded codeword or the network-coded bits instead.

A Jt-CNC algorithm for finding the XORed codeword was proposed in [13]. However, as will be discussed later, finding the ML XORed codeword requires exhaustive search that could have prohibitively high complexity. Therefore, the log-max approximation is adopted in [13] and the ML algorithm is simplified to (approximated with) a full-state Viterbi algorithm. The term "full-state" comes from the fact that this algorithm combines the trellises of both end nodes to make a virtual decoder. By searching the best path on the combined trellis with the Viterbi algorithm, [13] tries to decode the ML pair of codewords of the two end nodes. To further reduce the complexity, [13] simplifies the full-state Viterbi algorithm to a reduced-state Viterbi algorithm. Reference [13], however, did not benchmark their approximate algorithm with the optimal one. As we will show later, the algorithm proposed by us in this paper can yield better performance than that in [13].

In this paper, we aim to find the ML XORed bits within the codeword rather than the overall ML XORed codeword. In Section IV we show that our algorithm is ML XORed-bit optimal for synchronous PNC. Finding ML XORed bits turns out to have much lower complexity than finding the ML XORed codeword. This is quite different from the conventional point-to-point communication system, in which the simple Viterbi algorithm can be used to decode the ML codeword, and in which BCJR (slightly more complex than the Viterbi algorithm) can be used to decode the ML bits.

\section{B. Asynchronous PNC with Convolutional Codes}

In asynchronous PNC systems, the signals from the two end nodes may arrive at the relay with symbol misalignment and relative phase offset [5]. To our best knowledge, there was no Jt-CNC decoder for convolutional codes that can deal with integral-plus-fractional symbol misalignment. In [14], a convolutional decoding scheme with an XOR-CD algorithm was proposed to deal with integral symbol misalignment. As pointed out in [14], symbol misalignment entangles the channel-coded bits of the trellises of the two encoders in a way that ordinary Viterbi decoding, based on just one of the trellises, 
is not applicable. Therefore the XOR-CD algorithm for synchronous PNC cannot be applied anymore in the presence of integral symbol misalignment. Their solution is to rearrange the transmit order of the channel-coded bits into blocks, and pad $D_{\max }$ zeros between adjacent blocks. The zero padding acts as a guard interval between blocks that avoids the entanglement of channel-coded bits and facilitates Viterbi decoding. However, this scheme can only deal with integral symbol misalignment of at most $D_{\max }$ symbols. In addition, it incurs a code-rate loss factor of $\left(1-D_{\max } / L\right)$ due to the zero padding between blocks.

\section{Asynchronous PNC with Other Channel Codes}

The use of LDPC codes in asynchronous PNC systems have previously been considered. In [5], the authors designed a Jt-CNC decoder for the RA code that can deal with fractional symbol misalignment (i.e., symbol misalignment that is less than one symbol duration) and phase offset. Our decoding framework adopts the over-sampling technique proposed in [5] to address fractional symbol misalignment.

To deal with asynchrony in PNC, our decoding framework consists of three layers: symbol-realignment layer, codeword-realignment layer, and joint channel-decoding network coding (Jt-CNC) layer. The first two layers, symbol realignment and codeword realignment, counter fractional and integral symbol misalignments, respectively; the third layer, Jt-CNC, decodes the ML XORed bits. Other decoding schemes (e.g., XOR-CD, full-state Viterbi) can also be used in the third layer of the framework. We further show that our decoding framework is not only applicable when convolutional codes are adopted, it is also applicable when general cyclic codes are used. Besides convolutional codes, an important class of cyclic codes is the cyclic LDPC.

The Jt-CNC decoder proposed in [5] was extended by [15] to deal with general asynchrony using cyclic LDPC. However, the proposed decoder in [15] discards the non-overlapped part of the received signal, losing useful information that can potentially enhance performance. Therefore, for the decoder in [15], the larger the symbol misalignment, the worse the performance. By contrast, our framework makes full use of the non-overlapped portion of the signal so that the larger symbol misalignment can enhance performance.

\section{System Model}

We consider the application of PNC in a two-way relay network (TWRN) as shown in Fig. 11 In this model, nodes A and B exchange information with the help of relay node R. We assume that all nodes are half-duplex and there is no direct link between $\mathrm{A}$ and $\mathrm{B}$. 
With PNC, nodes A and B exchange one packet with each other in two time slots. The first time slot corresponds to an uplink phase, in which node A and node B transmit their channel-coded packets simultaneously to relay $\mathrm{R}$. The relay $\mathrm{R}$ then constructs a network-coded packet based on the simultaneously received signals from $\mathrm{A}$ and $\mathrm{B}$. This operation is referred to as the channel decoding network coding (CNC) process [10], because the received signals are decoded into a network-coded message rather than the individual messages from $\mathrm{A}$ and $\mathrm{B}$. The second time slot corresponds to a downlink phase, in which relay $\mathrm{R}$ channel-codes the network-coded message and broadcasts it to both $\mathrm{A}$ and $\mathrm{B}$. Upon receiving the network-coded packet, A (B) then attempts to recover the original packet transmitted by B (A) in the uplink phase using self-information [1]. This paper focuses on the design of the CNC algorithm in the uplink phase; the issue in the downlink phase is similar to that in conventional point-to-point transmission and does not require special treatment [10].

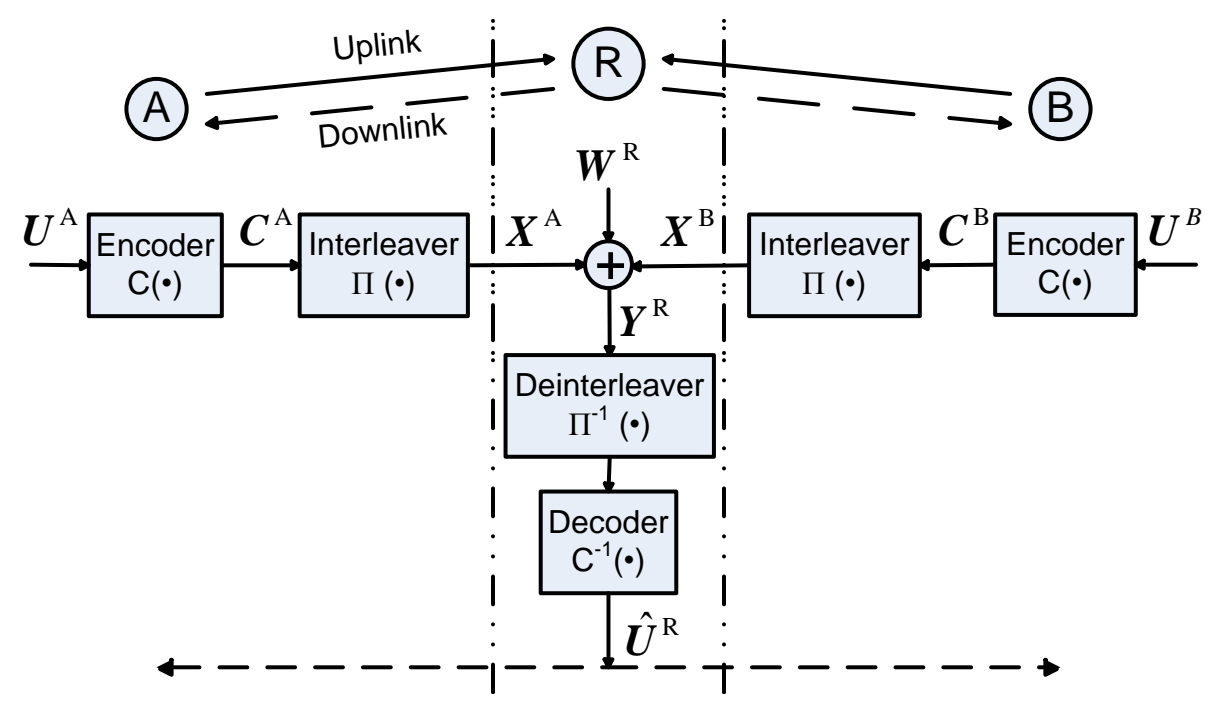

Fig. 1. System model of two-way relay network operated with physical-layer network coding.

$$
\text { cons }
$$$$
\text { (1) }
$$

$K$-bit binary sequences:

$$
U^{i}=\left(u_{1}^{i}, u_{2}^{i}, \cdots, u_{K}^{i}\right), i \in\{\mathrm{A}, \mathrm{B}\}
$$

\footnotetext{
${ }^{1}$ The use of other kinds of convolutional codes (e.g., zero-tailing and recursive) is discussed in the Appendix.
} 
where $u_{k}^{i}$ is the input bit of end nodes $i$ 's source packet at time $k$. The source packets are encoded into two $M$-bit channel-coded binary sequences. We assume nodes A and B use the same convolutional code with code rate $r=1 / R$ where $R$ is an integer. In the following presentation we choose $r=1 / 3$, thus $M=3 K$. The two channel-coded packets are

$$
\begin{aligned}
C^{i} & =\left(c_{1}^{i}, c_{2}^{i}, \cdots, c_{M}^{i}\right) \\
& =\left(\bar{c}_{1}^{i}, \bar{c}_{2}^{i}, \cdots, \bar{c}_{K}^{i}\right) \\
& =\left(c_{1,1}^{i}, c_{1,2}^{i}, c_{1,3}^{i}, c_{2,1}^{i}, c_{2,2}^{i}, \cdots, c_{K, 1}^{i}, c_{K, 2}^{i}, c_{K, 3}^{i}\right), i \in\{\mathrm{A}, \mathrm{B}\}
\end{aligned}
$$

where $c_{k, j}^{i}$ is the $j$ th channel-coded bit of end nodes $i$ 's channel-coded packet at time $k$; the 3-bit tuple $\bar{c}_{k}^{i}=\left(c_{k, 1}^{i}, c_{k, 2}^{i}, c_{k, 3}^{i}\right)$ is the output of the convolutional encoder of node $i$ at time $k$. Then, $C^{\mathrm{A}}$ and $C^{\mathrm{B}}$ are fed into block interleavers that realize the same permutation to produce

$$
\tilde{C}^{i}=\left(c_{1,1}^{i}, c_{2,1}^{i}, \cdots, c_{K, 1}^{i}, c_{1,2}^{i}, \cdots, c_{K, 2}^{i}, c_{1,3}^{i}, \cdots, c_{K, 3}^{i}\right), i \in\{\mathrm{A}, \mathrm{B}\} .
$$

Note that the permutation groups the $j$ th coded bits of all times together into a block. There are altogether three blocks. Finally, $\tilde{C}^{i}$ are modulated to produce the two sequences of $N$ complex symbols:

$$
X^{i}=\left(x_{1}^{i}, x_{2}^{i}, \cdots, x_{N}^{i}\right), i \in\{\mathrm{A}, \mathrm{B}\} .
$$

Throughout this paper, we focus on BPSK and QPSK modulations; our framework can be easily extended to higher order constellations [17]-[19]. For BPSK $N=3 K$ and $x_{n}^{i} \in\{1,-1\}$. For QPSK $N=3 K / 2$ and $x_{n}^{i} \in 1 / \sqrt{2}\{1+j,-1+j, 1-j,-1-j\}$. The complex symbol sequences $X^{\mathrm{A}}$ and $X^{\mathrm{B}}$ are shaped using a pulse shaping function $p(t)$ with symbol duration $T$ and transmitted. Without loss of generality, we assume $p(t)$ is the rectangular pulse throughout this paper.

Let us denote the channel coefficients of the channels from node A and node B to relay $\mathrm{R}$ by $h^{\mathrm{A}}$ and $h^{\mathrm{B}}$, respectively. Both $h^{\mathrm{A}}$ and $h^{\mathrm{B}}$ are complex numbers, whose phase difference $\phi=\angle\left(h^{\mathrm{B}} / h^{\mathrm{A}}\right)$ is the relative phase offset between node A and node B. We assume that the channel state information (CSI) $h^{\mathrm{A}}$ and $h^{\mathrm{B}}$ can be estimated at the relay $\mathrm{R}$ using orthogonal preambles [7].

The received complex baseband signal at the relay is

$$
y^{\mathrm{R}}(t)=\sum_{n=1}^{N}\left\{h^{\mathrm{A}} x_{n}^{\mathrm{A}} p(t-n T)+h^{\mathrm{B}} x_{n}^{\mathrm{B}} p(t-n T-\tau T)\right\}+w^{\mathrm{R}}(t)
$$

where $\tau T$ is the symbol misalignment (i.e., the arrival time of the signal of B lags the arrival time of the signal of A by $\tau T$ ), and $w^{\mathrm{R}}(t)$ is the noise, assumed to be circularly complex with variance $\sigma^{2}$. We 

1 as follows:

October 5, 2018

where

\section{Synchronous CONVOLUtional-CODED PNC}

This section focuses on synchronous convolutional-coded PNC, where the signals of node A and node B are symbol-aligned $(\tau=0)$. We first derive the XOR packet-optimal Jt-CNC algorithm that aims at finding the ML XORed source packet. We show that the XOR packet-optimal algorithm has prohibitively high complexity. Then we introduce our XOR bit-optimal Jt-CNC algorithm for finding the ML XORed bits, which has much lower complexity.

\section{A. XOR Packet-Optimal Decoding of Synchronous Convolutional-Coded PNC}

In the case of synchronous convolutional-coded PNC, the received baseband signal at relay $\mathrm{R}$ is obtained by setting symbol misalignment $\tau$ to zero in (5):

$$
y^{\mathrm{R}}(t)=\sum_{n=1}^{N}\left\{h^{\mathrm{A}} x_{n}^{\mathrm{A}} p(t-n T)+h^{\mathrm{B}} x_{n}^{\mathrm{B}} p(t-n T)\right\}+w^{\mathrm{R}}(t) .
$$

After matched filtering [5], the received baseband samples at relay $\mathrm{R}$ are

$$
Y^{\mathrm{R}}=\left(y_{1}^{\mathrm{R}}, y_{2}^{\mathrm{R}}, \cdots, y_{N}^{\mathrm{R}}\right)
$$

$$
y_{n}^{\mathrm{R}}=h^{\mathrm{A}} x_{n}^{\mathrm{A}}+h^{\mathrm{B}} x_{n}^{\mathrm{B}}+w_{n}^{\mathrm{R}} .
$$

The ML XORed source packet $\hat{U}^{\mathrm{R}}=\left(\hat{u}_{1}^{\mathrm{R}}, \hat{u}_{2}^{\mathrm{R}}, \cdots, \hat{u}_{K}^{\mathrm{R}}\right)$ (i.e., ML XOR of the source packets of node A and node B) is given by

$$
\hat{U}^{\mathrm{R}}=\arg \max _{U^{\mathrm{R}}} \sum_{U^{\mathrm{A}}, U^{\mathrm{B}}: U^{\mathrm{A}} \oplus U^{\mathrm{B}}=U^{\mathrm{R}}} \exp \left(-\mathcal{M}\left(X^{\mathrm{A}}, X^{\mathrm{B}}\right)\right)
$$

where $\oplus$ denotes the binary bit-wise XOR operator; $X^{\mathrm{A}}$ and $X^{\mathrm{B}}$ are the convolutional-encoded and modulated baseband signal of $U^{\mathrm{A}}$ and $U^{\mathrm{B}}$, respectively; and $\mathcal{M}\left(X^{\mathrm{A}}, X^{\mathrm{B}}\right)$ is the distance metric defined

$$
\begin{aligned}
\mathcal{M}\left(X^{\mathrm{A}}, X^{\mathrm{B}}\right) & =\sum_{n=1}^{N} \frac{\left|y_{n}^{\mathrm{R}}-h^{\mathrm{A}} x_{n}^{\mathrm{A}}-h^{\mathrm{B}} x_{n}^{\mathrm{B}}\right|^{2}}{2 \sigma^{2}} \\
& =\frac{\left\|Y^{\mathrm{R}}-h^{\mathrm{A}} X^{\mathrm{A}}-h^{\mathrm{B}} X^{\mathrm{B}}\right\|_{2}^{2}}{2 \sigma^{2}} .
\end{aligned}
$$


For source packets $U^{\mathrm{A}}$ and $U^{\mathrm{B}}$ of length $K$, the functional mapping from $U^{\mathrm{A}}$ and $U^{\mathrm{B}}$ to the XORed source packet $U^{\mathrm{R}}$ can be expressed as

$$
f_{\text {packet }}:\{0,1\}^{K} \times\{0,1\}^{K} \rightarrow\{0,1\}^{K} \text {. }
$$

The mapping in $(11)$ is a $2^{K}$-to- 1 mapping; that is, there are $2^{K}$ possible $\left(U^{\mathrm{A}}, U^{\mathrm{B}}\right)$ that can produce a particular $U^{\mathrm{R}}$. This is where the complexity lies in 9 . For each $X^{\mathrm{R}}$, the baseband channel-coded signal corresponding to $U^{\mathrm{R}}$, we need to examine $2^{K}$ possible combinations of $X^{\mathrm{A}}$ and $X^{\mathrm{B}}$. The Viterbi algorithm is a shortest-path algorithm that computes a path in the trellis of $\left(U^{\mathrm{A}}, U^{\mathrm{B}}\right)$. Meanwhile, each $U^{\mathrm{R}}$ is associated with $2^{K}$ paths in the trellis. There is no known exact computation method for 9 ) except to exhaustively sum over the possible combinations of $\left(U^{\mathrm{A}}, U^{\mathrm{B}}\right)$ for each $U^{\mathrm{R}}$.

For each possible $\left(U^{\mathrm{A}}, U^{\mathrm{B}}\right)$, we need to sum over $N$ terms in 10 to compute $\mathcal{M}\left(X^{\mathrm{A}}, X^{\mathrm{B}}\right)$. For a code-rate $r$ code and M-QAM modulation, $N=K /\left[r \log _{2}(M)\right]$. Computing each term in (10) takes two complex operations, and the summation takes $(N-1)$ operations. Hence the complexity of one combination of $\left(U^{\mathrm{A}}, U^{\mathrm{B}}\right)$ is $\left(3 K /\left[r \log _{2}(M)\right]-1\right)$. Moreover, to find the maximum of $(9),\left(2^{K}-1\right)$ comparisons are needed. Given that there are $2^{K}$ possible $U^{\mathrm{R}}$, from which we want to find the optimal $\hat{U}^{\mathrm{R}}$, the overall complexity is therefore $2^{2 K}\left(3 K /\left[r \log _{2}(M)\right]-1\right)+2^{K}-1$. In Big-O notation, the complexity is $O\left(K 2^{2 K}\right)$.

This is a big contrast with the regular point-to-point communication system, in which the Viterbi algorithm used to find the ML codeword is of polynomial complexity only. For PNC systems, the complexity of XOR packet-optimal decoding algorithm is exponential with $K$, the length of source packet.

\section{B. XOR Bit-Optimal Decoding of Synchronous Convolutional-Coded PNC}

To reduce complexity, we consider an XOR bit-optimal Jt-CNC decoder based on the framework of Belief Propagation (BP) algorithms. The proposed decoder aims to find the ML XORed source bit rather than the ML XORed source packet. We give two important results: (i) the proposed Jt-CNC decoder is optimal in terms of BER performance; and (ii) the complexity is linear in packet length $K$.

Unlike finding ML XORed packets, for which the Viterbi algorithm is of little use, the BP (BCJR) algorithm can find the ML XORed source bit readily without incurring exponential growth in complexity. We first explain the reason before describing the BP algorithm in detail. 
The $k$ th ML XORed source bits $\hat{u}_{k}^{\mathrm{R}}, k=1,2, \ldots K$ is given by

$$
\hat{u}_{k}^{\mathrm{R}}=\arg \max _{u_{k}^{\mathrm{R}}} \sum_{\bar{u}_{k}: u_{k}^{\mathrm{A} \oplus u_{k}^{\mathrm{B}}=u_{k}^{\mathrm{R}}}} \operatorname{Pr}\left(\bar{u}_{k} \mid Y^{\mathrm{R}}\right)
$$

where $\operatorname{Pr}\left(\bar{u}_{k} \mid Y^{\mathrm{R}}\right)$ can be calculated using the BP algorithm. Fortunately, finding the ML XORed bits in PNC systems has much lower complexity, because the functional mapping from $\left(u_{k}^{\mathrm{A}}, u_{k}^{\mathrm{B}}\right)$ to $u_{k}^{\mathrm{R}}$ can be expressed as

$$
f_{\text {bit }}:\{0,1\} \times\{0,1\} \rightarrow\{0,1\} .
$$

The mapping in (13) is a 2-to-1 mapping; hence for each possible XOR bit we need to examine only two pairs of source bits. Importantly, the $\mathrm{BP}$ algorithm can compute $\operatorname{Pr}\left(u_{k}^{\mathrm{A}}, u_{k}^{\mathrm{B}} \mid Y^{\mathrm{R}}\right)$ easily, from which $\operatorname{Pr}\left(u_{k}^{\mathrm{A}} \oplus u_{k}^{\mathrm{B}} \mid Y^{\mathrm{R}}\right)$ can readily be obtained through the 2-to-1 mapping. Because finding a single ML XORed source bit in (12) takes two summations and one comparison, so finding all the ML XORed source bits takes $3 K$ operations beyond the operations by the $\mathrm{BP}$ algorithm that computes $\operatorname{Pr}\left(u_{k}^{\mathrm{A}}, u_{k}^{\mathrm{B}} \mid Y^{\mathrm{R}}\right), k=$ $1,2, \ldots K$.

As will be elaborated later in this section, the BP algorithm has three steps. The initialization takes $9 K /\left[r \log _{2}(M)\right]$ operations in 16 . The forward/backword recursions take $6 \cdot 2^{2 / r} K S^{2}$ operations where $S$ is the number of decoder's states. The termination takes $4 \cdot 2^{2 / r} K S^{2}$ operations. Therefore finding the ML XORed source bits of length- $K$ packets has an overall complexity of $9 K /\left[r \log _{2}(M)\right]+6 \cdot 2^{2 / r} K S^{2}+$ $4 \cdot 2^{2 / r} K S^{2}+3 K$. In Big-O notation, the complexity of source bit-optimal decoding algorithm is $O(K)$. We next elaborate the bit-optimal Jt-CNC algorithm.

$\mathrm{BP}$ is a framework for generating inference-making algorithms for graphical models, in which there are two kinds of nodes: variable nodes and factor nodes. Each variable node represents a variable, such as the state variable of the convolutional encoder; each factor node indicates the relationship among all variable nodes connected to it. For example the state transition function of a convolutional encoder is represented by a factor node. The goal of BP is to compute the marginal probability distributions $\operatorname{Pr}\left(u_{k}^{\mathrm{A}}, u_{k}^{\mathrm{B}} \mid Y^{\mathrm{R}}\right)$ for all $k$. This goal is achieved by means of a sum-product message-passing algorithm [20].

Fig. 2 shows the Tanner graph of our bit-optimal Jt-CNC decoder. Unlike the conventional point-to-point convolutional decoder for single-user systems with only one transmitter, the Jt-CNC decoder combines the states and the trellis of both transmitters A and B. In Fig. 2, vectors $S=\left(s_{0}, s_{1}, \cdots, s_{K}\right)$ represents the state variables, where state $s_{k}$ combines the state of both end nodes' states; vector $U=\left(\bar{u}_{1}, \bar{u}_{2}, \cdots, \bar{u}_{K}\right)$, where $\bar{u}_{k}=\left(u_{k}^{\mathrm{A}}, u_{k}^{\mathrm{B}}\right)$, represents the "virtual" source packet consisting of the duple of the two source packets from nodes $\mathrm{A}$ and $\mathrm{B}$; similarly, vector $C=\left(\bar{c}_{1}, \bar{c}_{2}, \cdots, \bar{c}_{K}\right)$, where $\bar{c}_{k}=\left(\bar{c}_{k}^{\mathrm{A}}, \bar{c}_{k}^{\mathrm{B}}\right)$ (as defined in 


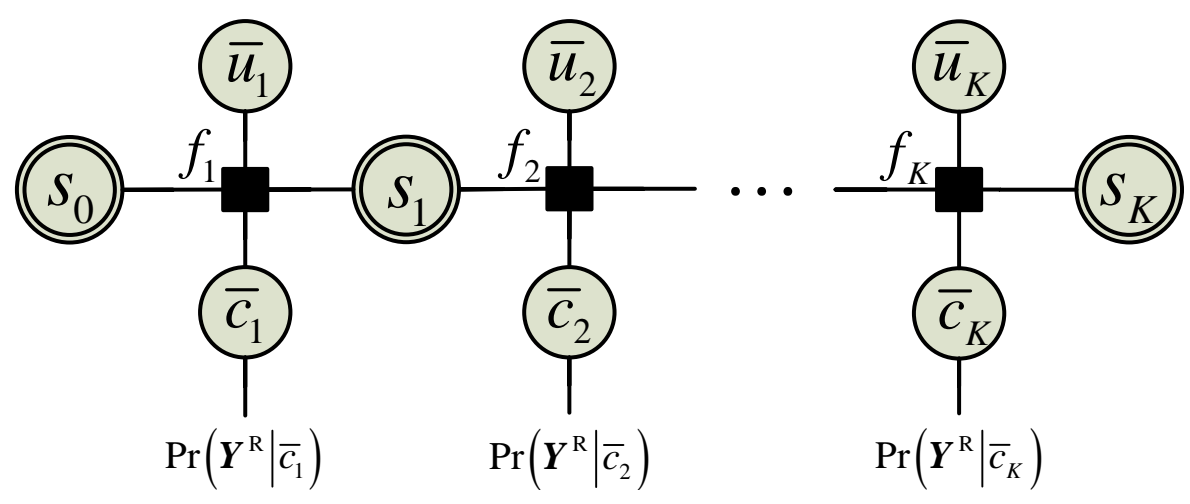

Fig. 2. Tanner graph of the Jt-CNC decoder on which the BP algorithm operates: $s_{k}$ is the state variable, $\bar{u}_{k}$ is the source bit, $\bar{c}_{k}$ is the channel-coded bit; $f_{i}$ is the factor node that represents the state transition function of the Jt-CNC decoder. 7 probability (APP) $\operatorname{Pr}\left(\bar{u}_{k} \mid Y^{\mathrm{R}}\right)$ by

$$
\operatorname{Pr}\left(u_{k}^{\mathrm{R}} \mid Y^{\mathrm{R}}\right)=\max _{u_{k}^{\mathrm{R}}} \sum_{\bar{u}_{k}: u_{k}^{\mathrm{A}} \oplus u_{k}^{\mathrm{B}}=u_{k}^{\mathrm{R}}} \operatorname{Pr}\left(\bar{u}_{k} \mid Y^{\mathrm{R}}\right)
$$

2. $\bar{c}_{k}^{i}$ denotes the group of channel-coded bits of node $i$ at time $k$ ), represents the "virtual" channel-coded packet, assuming that both nodes A and B use the same channel code. The behavior of the decoder is defined by the functions of the factors node $f_{k}\left(s_{k-1}, \bar{u}_{k}, \bar{c}_{k}, s_{k}\right)$ that represents the state transition rule of the trellis.

The goal of the Jt-CNC decoder is to find the maximum likelihood XOR bit $u_{k}^{\mathrm{R}}$ through the a posteriori where $\operatorname{Pr}\left(\bar{u}_{k} \mid Y^{\mathrm{R}}\right)$ can be computed exactly by the sum-product message-passing algorithm thanks to the tree structure of the Tanner graph associated with convolutional nodes [21]. The sum-product algorithm, when applied to decode convolutional codes, is the well-known BCJR algorithm [12]. The difference in our situation here is that instead of the source bit from one source, we are decoding for the bit duple $\bar{u}_{k}=\left(u_{k}^{\mathrm{A}}, u_{k}^{\mathrm{B}}\right)$ from the two sources.

We now explain the sum-product algorithm in detail. Fig. 3 depicts the messages being passed around a factor node within the overall Tanner graph of Fig. 2. We follow the notation of the original paper on the BCJR algorithm [12]. In the forward direction, the message from $s_{k-1}$ to $f_{k}$ is denoted by $\alpha\left(s_{k-1}\right)$, and the message from $f_{k}$ to $s_{k}$ is denoted by $\alpha\left(s_{k}\right)$. In the backward direction, the message from $s_{k}$ to $f_{k}$ is denoted by $\beta\left(s_{k}\right)$, and the message from $f_{k}$ to $s_{k-1}$ is denoted by $\beta\left(s_{k-1}\right)$. Additionally, $\gamma\left(\bar{c}_{k}\right)$ denotes the message from $\bar{c}_{k}$ to $f_{k}$, and $\delta\left(\bar{u}_{k}\right)$ denotes the message from $f_{k}$ to $\bar{u}_{k}$. Note that $\delta\left(\bar{u}_{k}\right)$ is the APP $\operatorname{Pr}\left(\bar{u}_{k} \mid Y^{\mathrm{R}}\right)$ and the goal here is to compute it.

Since the Tanner graph of the Jt-CNC decoder is cycle-free, the operation of the sum-product algorithm 


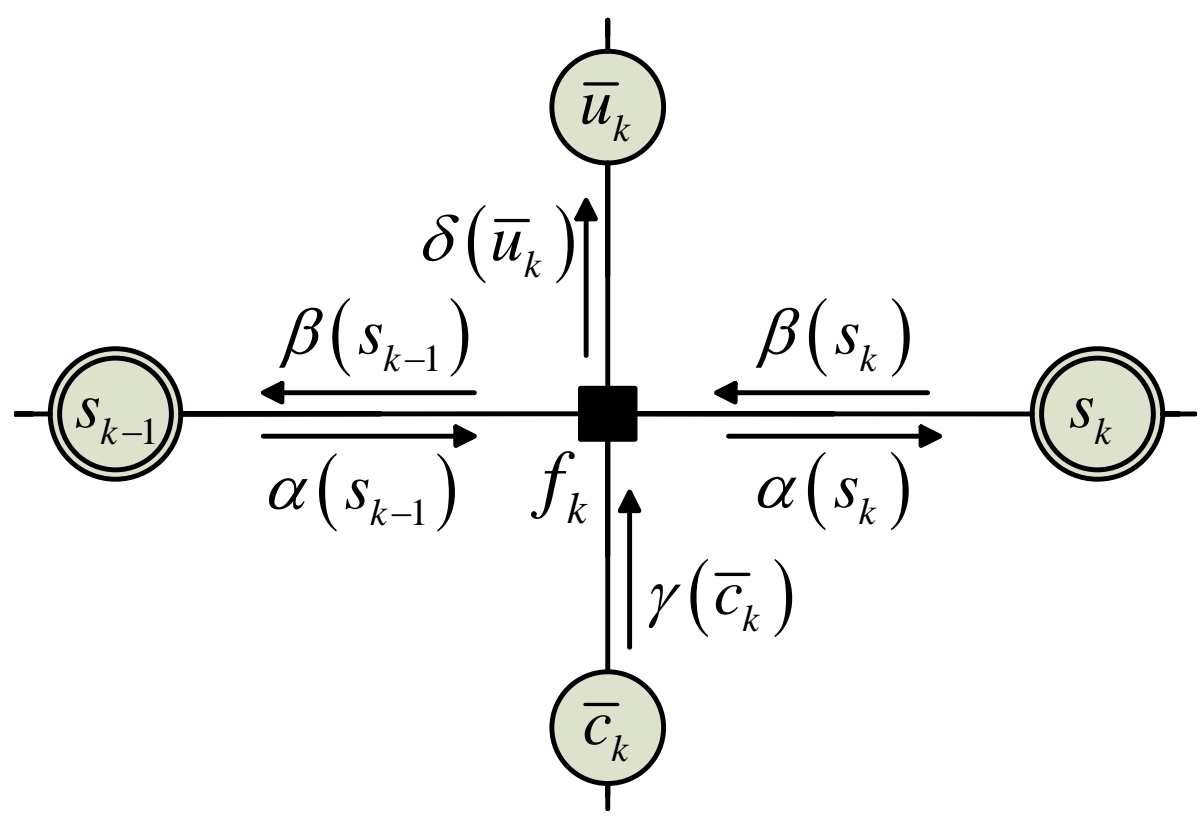

Fig. 3. The messages being passed around a factor node during the operation of the sum-product algorithm.

Initialization As usual in a cycle-free Tanner graph, the sum-product algorithm begins at the leaf nodes. Since tail biting convolutional code is used, the initial and terminal states of end node's convolutional encoders are the same, and they are decided by the random input message. Therefore the initial and terminal states are uniformly distributed among all possible states, so the message $\alpha\left(s_{0}\right)$ and $\beta\left(s_{K}\right)$ are initialized as

$$
\alpha\left(s_{0}\right)=\frac{1}{N_{S}}, \forall s_{0}
$$

and

$$
\beta\left(s_{K}\right)=\frac{1}{N_{S}}, \forall s_{K}
$$

1 where $N_{S}$ is the number of states per stage. 
The message $\gamma\left(\bar{c}_{k}\right)$ is the likelihood function of $\bar{c}_{k}$ based on the evidence $Y^{\mathrm{R}}$. For example, if the code rate is $1 / 3$ and the BPSK modulation is used, then $\bar{c}_{k}=\left(c_{k, 1}^{\mathrm{A}}, c_{k, 2}^{\mathrm{A}}, c_{k, 3}^{\mathrm{A}}, c_{k, 1}^{\mathrm{B}}, c_{k, 2}^{\mathrm{B}}, c_{k, 3}^{\mathrm{B}}\right)$. These channelcoded bits $\bar{c}_{k}$ are mapped to BPSK modulated symbols $\left(x_{3 k-2}^{\mathrm{A}}, x_{3 k-1}^{\mathrm{A}}, x_{3 k}^{\mathrm{A}}\right)$ and $\left(x_{3 k-2}^{\mathrm{B}}, x_{3 k-1}^{\mathrm{B}}, x_{3 k}^{\mathrm{B}}\right)$ at node A and node $\mathrm{B}$, respectively. Given the overlapped signal $Y^{\mathrm{R}}$ at the relay node, the likelihood of $\bar{c}_{k}$ is calculated by

$$
\gamma\left(\bar{c}_{k}\right)=\operatorname{Pr}\left(Y^{\mathrm{R}} \mid \bar{c}_{k}\right)=\prod_{j=1}^{3} \frac{1}{\sqrt{2 \pi \sigma^{2}}} \exp \left\{-\frac{\left|y_{3(k-1)+j}^{\mathrm{R}}-h^{\mathrm{A}} x_{3(k-1)+j}^{\mathrm{A}}-h^{\mathrm{B}} x_{3(k-1)+j}^{\mathrm{B}}\right|^{2}}{2 \sigma^{2}}\right\} .
$$

Forward/backward recursion After initializing the messages from leaf nodes, we can compute the message $\alpha\left(s_{k}\right)$ and $\beta\left(s_{k}\right)$ recursively by following the message update rule below [21]:

$$
\begin{aligned}
\alpha\left(s_{k}\right) & =\sum_{s_{k-1}, \bar{u}_{k}, \bar{c}_{k}} f_{k}\left(s_{k-1}, \bar{u}_{k}, \bar{c}_{k}, s_{k}\right) \alpha\left(s_{k-1}\right) \gamma\left(\bar{c}_{k}\right) \\
\beta\left(s_{k-1}\right) & =\sum_{s_{k}, \bar{u}_{k}, \bar{c}_{k}} f_{k}\left(s_{k-1}, \bar{u}_{k}, \bar{c}_{k}, s_{k}\right) \beta\left(s_{k}\right) \gamma\left(\bar{c}_{k}\right) .
\end{aligned}
$$

Termination In the final step, the algorithm terminates with the computation of $\delta\left(\bar{u}_{k}\right)$, which gives the APP of the source bit $\bar{u}_{k}$.

$$
\delta\left(\bar{u}_{k}\right)=\sum_{s_{k-1}, s_{k}, \bar{c}_{k}} f_{k}\left(s_{k-1}, \bar{u}_{k}, \bar{c}_{k}, s_{k}\right) \alpha\left(s_{k-1}\right) \gamma\left(\bar{c}_{k}\right) \beta\left(s_{k}\right) .
$$

The summation in $\left[18\right.$ is over different trellis transitions $e=\left(s_{k-1}, \bar{u}_{k}, \bar{c}_{k}, s_{k}\right)$ with fixed $\bar{u}_{k}$, such that $f_{k}(e)=1$ if $e$ is a valid transition, and $f_{k}(e)=0$ otherwise. For example, if input $\bar{u}_{k}$ causes a state transition from $s_{k-1}$ to $s_{k}$ and the output is $\bar{c}_{k}$, then $f_{k}(e)=1$; on the other hand, if input $\bar{u}_{k}$ causes a state transition from $s_{k-1}$ to a state not equal to $s_{k}$ or the output is not $\bar{c}_{k}$, then $f_{k}(e)=0$.

\section{ASYNCHRONOUS CONVOLUTIONAL-CODED PNC}

In this section, we present our three-layer decoding framework for asynchronous convolutional-coded PNC. The asynchrony causes unique challenges that the synchronous decoder in Section IV cannot handle. As shown in Fig. 4, when the signals of nodes A and B arrive at the relay at different times, their symbols can be misaligned. The symbol misalignment consists of two parts: an integral part $\tau_{\mathrm{I}}$ and a fractional part $\tau_{\mathrm{F}}$. These two components impose different challenges: the fractional symbol misalignment causes overlaps of adjacent symbols so that the symbol-boundary preserving sampling as expressed in (8) is no more valid; the integral symbol misalignment entangles the channel-coded bits of nodes A and B in such a way that the decoding scheme as proposed in Section IV cannot be applied anymore.

To address these challenges, together with the Jt-CNC decoder, we add two layers to construct an integrated framework illustrated in Fig. 5. First, to address the fractional symbol misalignment, the 


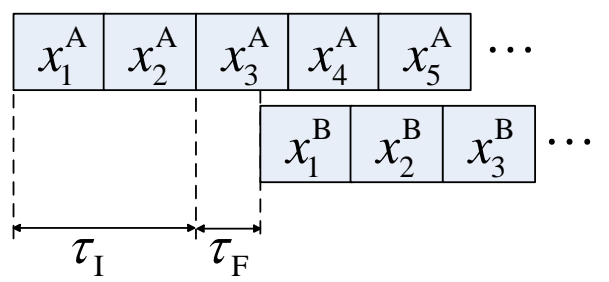

Fig. 4. Symbol misalignment in PNC: a general symbol misalignment consists of an integral part $\tau_{\mathrm{I}}=2$ and a fractional part $\tau_{\mathrm{F}}=0.7$.

symbol-realignment layer uses a BP algorithm at the relay to "realign" the soft information of the symbols. Second, the codeword-realignment layer uses an interleaver/deinterleaver set-up to accommodate the integral symbol misalignment. As a result, the three-layer decoding framework can deal with the integral-plus-fractional symbol misalignment.

Furthermore, building on the insight obtained from our study of convolutional-coded PNC, we propose an algorithm that can deal with general symbol misalignment with cyclic codes. That is, our decoding framework can incorporate not just convolutional codes, but all cyclic codes to address the challenges in asynchronous PNC.

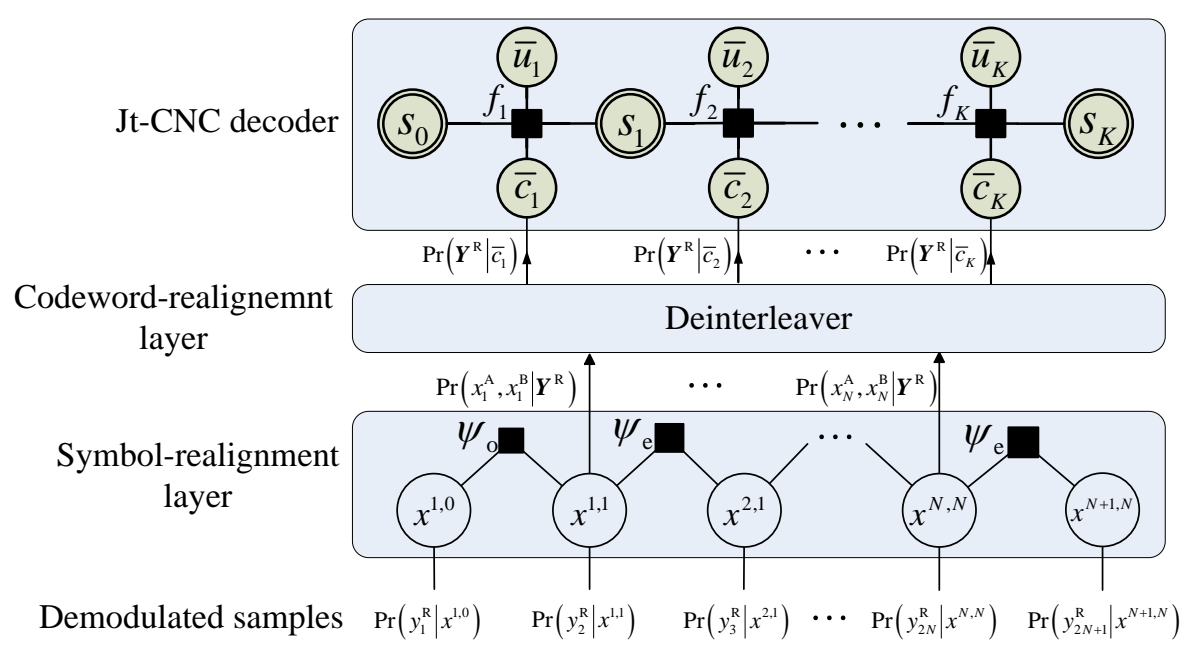

Fig. 5. Decoding framework for asynchronous convolutional-coded PNC systems. This framework can deal with an integralplus-fractional symbol misalignment and is BER-optimal. 


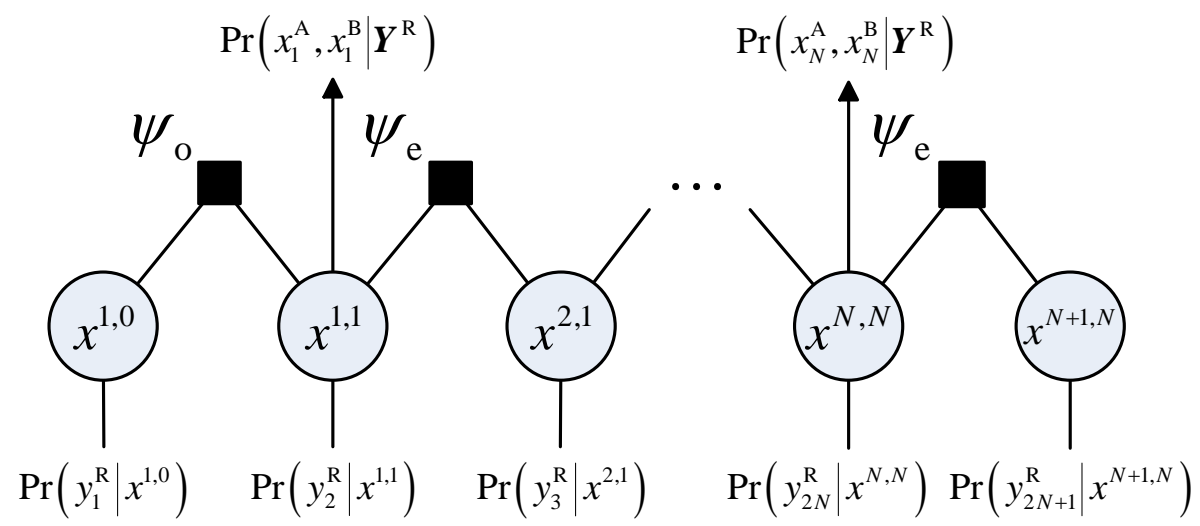

Fig. 6. Tanner graph of the symbol-realignment layer. $x^{i, j}$, denotes the joint symbol $\left(x_{i}^{\mathrm{A}}, x_{j}^{\mathrm{B}}\right)$, are the variable nodes; $\psi_{\mathrm{o}}$ and $\psi_{\mathrm{e}}$ are the factor nodes that indicates the compatibility associated with the variable nodes; The likelihood probabilities $\operatorname{Pr}\left(y_{2 n-1}^{\mathrm{R}} \mid x^{n, n-1}\right)$ and $\operatorname{Pr}\left(y_{2 n}^{\mathrm{R}} \mid x^{n, n}\right)$ are the evidences from observation $Y^{\mathrm{R}}$.

\section{A. Symbol-Realignment Layer: Addressing Fractional Symbol Misalignment}

For simplicity, as in [5], we assume the use of rectangular pulse to carry the modulated signal, and the use of the doubling sampling technique to obtain two samples per symbol period. Let us first ignore the integral part of symbol misalignment and only consider the fractional part (i.e., $\tau<1$ ). Furthermore, let us assume $\left|h_{\mathrm{A}}\right|=\left|h_{\mathrm{B}}\right|=\sqrt{P}$ where $P$ is the transmission power of end nodes.

With double sampling on the received signal $y^{\mathrm{R}}(t)$, the total number of samples obtained per frame is $2 N+1$, where $N$ is the number of symbols per frame (for both users $\mathrm{A}$ and $\mathrm{B}$ ). The relay uses the $2 N+1$ samples to compute the soft information $\operatorname{Pr}\left(x_{n}^{\mathrm{A}}, x_{n}^{\mathrm{B}} \mid Y^{\mathrm{R}}\right)$, where instead of the expression in 7), $Y^{\mathrm{R}}=\left(y_{1}^{\mathrm{R}}, y_{2}^{\mathrm{R}}, \cdots, y_{2 N+1}^{\mathrm{R}}\right)$ consists of the $2 N+1$ samples. Thus, as far as the soft information is concerned, the fractional symbol misalignment is removed and the symbols are realigned. We emphasize that this realignment of soft information is a key step. Once that is done, the channel decoding algorithm for synchronous PNC as proposed in Section IV can be applied.

We can write the samples obtained at the relay $\mathrm{R}$ as follows (after normalization):

$$
\begin{gathered}
y_{2 n-1}^{\mathrm{R}}=x_{n}^{\mathrm{A}}+x_{n-1}^{\mathrm{B}} e^{j \varphi}+w_{2 n-1}^{\mathrm{R}} \\
y_{2 n}^{\mathrm{R}}=x_{n}^{\mathrm{A}}+x_{n}^{\mathrm{B}} e^{j \varphi}+w_{2 n}^{\mathrm{R}}
\end{gathered}
$$

where $n=1,2, \ldots, N, x_{0}^{\mathrm{B}}=0$ and $y_{2 N+1}^{\mathrm{R}}=x_{N}^{\mathrm{B}} e^{j \varphi}+w_{2 N+1}^{\mathrm{R}}$. The terms $w_{2 n-1}^{\mathrm{R}}$ and $w_{2 n}^{\mathrm{R}}$ are zero-mean complex Gaussian noise with variances $\sigma^{2} / \tau P$ and $\sigma^{2} /(1-\tau) P$ per dimension, respectively.

We use a BP algorithm to compute soft information of $\operatorname{Pr}\left(x_{n}^{\mathrm{A}}, x_{n}^{\mathrm{B}} \mid Y^{\mathrm{R}}\right)$ from the $2 N+1$ samples. The 
associated Tanner graph is shown in Fig. 6. In the Tanner graph $x^{i, j} \triangleq\left(x_{i}^{\mathrm{A}}, x_{j}^{\mathrm{B}}\right)$ are the variable node; $\psi_{\mathrm{o}}$ and $\psi_{\mathrm{e}}$ are the compatibility functions associated with the factor nodes. The compatibility functions model the correlation between two adjacent symbols and are defined as

$$
\begin{aligned}
& \psi_{\mathrm{o}}\left(x^{n, n-1}, x^{n, n}\right)= \begin{cases}1 & \text { if the values of } x_{n}^{\mathrm{A}} \text { in } x^{n, n-1} \text { and } x^{n, n} \text { are equal } \\
0 & \text { otherwise }\end{cases} \\
& \psi_{\mathrm{e}}\left(x^{n, n}, x^{n+1, n}\right)= \begin{cases}1 & \text { if the values of } x_{n}^{\mathrm{B}} \text { in } x^{n, n} \text { and } x^{n+1, n} \text { are equal } \\
0 & \text { otherwise }\end{cases}
\end{aligned}
$$

The likelihood probabilities $\operatorname{Pr}\left(y_{2 n-1}^{\mathrm{R}} \mid x^{n, n-1}\right)$ and $\operatorname{Pr}\left(y_{2 n}^{\mathrm{R}} \mid x^{n, n}\right)$ are the evidences from observation $Y^{\mathrm{R}}$. The computation of these evidences is given by

$$
\operatorname{Pr}\left(y_{2 n-1}^{\mathrm{R}} \mid x^{n, n-1}\right)=\operatorname{Pr}\left(y_{2 n-1}^{\mathrm{R}} \mid x_{n}^{\mathrm{A}}, x_{n-1}^{\mathrm{B}}\right)=\frac{1}{\sqrt{2 \pi \sigma^{2} / \tau}} \exp \left\{-\frac{\left|y_{2 n-1}^{\mathrm{R}}-x_{n}^{\mathrm{A}}-x_{n-1}^{\mathrm{B}}\right|^{2}}{2 \sigma^{2} / \tau}\right\}
$$

and

$$
\operatorname{Pr}\left(y_{2 n}^{\mathrm{R}} \mid x^{n, n}\right)=\quad \operatorname{Pr}\left(y_{2 n}^{\mathrm{R}} \mid x_{n}^{\mathrm{A}}, x_{n}^{\mathrm{B}}\right)=\frac{1}{\sqrt{2 \pi \sigma^{2} /(1-\tau)}} \exp \left\{-\frac{\left|y_{2 n}^{\mathrm{R}}-x_{n}^{\mathrm{A}}-x_{n}^{\mathrm{B}}\right|^{2}}{2 \sigma^{2} /(1-\tau)}\right\}
$$

Given the evidences computed in $21 \mathrm{a}$ ) and $(21 \mathrm{~b})$, the message update equations can be derived using the standard sum-product formula of BP [21]. Note that the Tanner graph has a tree structure. This means that the BP algorithm can compute the exact APP of $x^{n, n}$ and $x^{n, n-1}$ for $n=1, \cdots, N$. Furthermore, the solution can be found by passing the messages only once in each direction of the Tanner graph. Although we can compute the APP of all variable nodes, we only use the APP of $\operatorname{Pr}\left(x_{n}^{\mathrm{A}}, x_{n}^{\mathrm{B}} \mid Y^{\mathrm{R}}\right)$ for further decoding.

\section{B. Codeword-Realignment Layer: Countering Integral Symbol Misalignment}

Since the fractional part of symbol misalignment has been removed in the symbol-realignment layer, here we only consider the integral part of symbol misalignment in this subsection. Recall that in Section IV we used (16) to compute the message $\gamma\left(\bar{c}_{k}\right)$. Equation $[16$ requires that the modulated symbols of end nodes $\mathrm{A}$ and $\mathrm{B}$ are symbol-by-symbol aligned (i.e., $x_{n}^{\mathrm{A}}$ must align with $x_{n}^{\mathrm{B}}$ ). However, with integral symbol misalignment $\tau_{\mathrm{I}}, x_{n}^{\mathrm{A}}$ will be aligned with $x_{n-\tau_{\mathrm{I}}}^{\mathrm{B}}$ therefore the algorithm proposed in Section IV becomes invalid. 
The codeword-realignment layer addresses this challenge using a specially designed interleaver/deinterleaver at the end/relay nodes. At the end nodes, we use the same block interleaver with $R$ rows and $M / R$ columns, where $r=1 / R$ is the code rate and $M$ is the number of bits in the codeword. To interleave, the channel-coded bits are filled into the interleaver column-wise, and read out row-wise. Then the interleaved packets are modulated and transmitted simultaneously to the relay. Upon receiving the overlapped signal (with symbol misalignment), the relay first deals with the fractional symbol misalignment with the algorithm proposed in Section $\mathrm{V}-\mathrm{A}$. Then the relay uses the same block deinterleaver to deinterleave the received signal.

Let us consider an example with code rate $1 / 3$ convolutional code, BPSK modulation, and integral symbol misalignment $\tau=2$ (in this subsection, we only consider the integral part of $\tau$ ). As specified in Section III the channel-coded packets of node $\mathrm{A}$ and node $\mathrm{B}$ are $C^{\mathrm{A}}$ and $C^{\mathrm{B}}$, respectively. Then the channel-coded packet is bit-interleaved with a block interleaver with $M / R$ rows and $R$ columns. The interleaved packets $\tilde{C}^{i}, i \in \mathrm{A}, \mathrm{B}$ are BPSK modulated to produce the transmitted signal

$$
X^{i}=\left(x_{1,1}^{i}, x_{2,1}^{i}, \cdots, x_{K, 1}^{i}, \quad x_{1,2}^{i}, \cdots, x_{K, 2}^{i}, x_{1,3}^{i}, \cdots, x_{K, 3}^{i}\right), i \in\{\mathrm{A}, \mathrm{B}\}
$$

where $x_{k, l}^{i}=1-2 c_{k, l}^{i}$, and $c_{k, l}^{i}$ is defined in 2 . The received signal samples will be the superposition of the following two sequences

$$
\begin{array}{rllllllll}
x_{1,1}^{\mathrm{A}} \quad x_{2,1}^{\mathrm{A}} & x_{3,1}^{\mathrm{A}} & x_{4,1}^{\mathrm{A}} & x_{5,1}^{\mathrm{A}} & \cdots & x_{K, 3}^{\mathrm{A}} & \\
& & + & & & \\
& & x_{1,1}^{\mathrm{B}} & x_{2,1}^{\mathrm{B}} & x_{3,1}^{\mathrm{B}} & \cdots & x_{K-2,3}^{\mathrm{B}} & x_{K-1,3}^{\mathrm{B}} & x_{K, 3}^{\mathrm{B}} .
\end{array}
$$

The relay first aligns the unoverlapped (clear) part of signal: $x_{1,1}^{\mathrm{A}} x_{2,1}^{\mathrm{A}}$ at the head and $x_{K-1,3}^{\mathrm{B}} x_{K, 3}^{\mathrm{B}}$ at the tail. Then the relay deinterleaves this packet to restore node A's transmission order. After deinterleaving, the received packet becomes the superposition of the following sequences

$$
\begin{array}{cccccccccc}
x_{1,1}^{\mathrm{A}} & x_{1,2}^{\mathrm{A}} & x_{1,3}^{\mathrm{A}} & x_{2,1}^{\mathrm{A}} & x_{2,2}^{\mathrm{A}} & x_{2,3}^{\mathrm{A}} & \cdots & x_{K, 1}^{\mathrm{A}} & x_{K, 2}^{\mathrm{A}} & x_{K, 3}^{\mathrm{A}} \\
& & & & & & \\
x_{K-1,3}^{\mathrm{B}} & x_{K-1,1}^{\mathrm{B}} & x_{K-1,2}^{\mathrm{B}} & x_{K, 3}^{\mathrm{B}} & x_{K, 1}^{\mathrm{B}} & x_{K, 2}^{\mathrm{B}} & \cdots & x_{K-2,1}^{\mathrm{B}} & x_{K-2,2}^{\mathrm{B}} & x_{K-2,3}^{\mathrm{B}} .
\end{array}
$$

The signal in 24 is equivalent to the superposition of modulated signals of $C^{\mathrm{A}}$ and $C_{(6)}^{\mathrm{B}}$, where $C_{(6)}^{\mathrm{B}}$ denotes the 6 bits right circular-shifted version of $C^{\mathrm{B}}$. Since tail biting convolutional code with code rate $1 / R$ is quasi-cyclic with period $R, C_{(\tau R)}^{\mathrm{B}}$, the $\tau R$ bit circular-shifted version of $C^{\mathrm{B}}$, is also a valid codeword [16], [22], [23]. Hence we can apply the Jt-CNC decoding algorithm proposed in Section IV] Furthermore, as we prove in the Appendix, a very good property of convolutional code is: the source 
packet corresponding to $C_{(\tau R)}^{\mathrm{B}}$ is $U_{(\tau)}^{\mathrm{B}}$, the $\tau$-bit right circular-shifted version of node B's source packet $U^{\mathrm{B}}$.

As a result, in the presence of integral symbol misalignment, our bit-optimal decoding algorithm will output $U^{\mathrm{R}}=U^{\mathrm{A}} \oplus U_{(\tau)}^{\mathrm{B}}$. However, node A (B) can still restore the information of node B (A). Node A can first XOR $U^{\mathrm{R}}$ with its own packet to obtain $U_{(\tau)}^{\mathrm{B}}$, then left shift it $\tau$ bits to restore $U^{\mathrm{B}}$. Node B can first right shift its own packet to obtain $U_{(\tau)}^{\mathrm{B}}$ and then XOR it with $U^{\mathrm{R}}$ to obtain $U^{\mathrm{A}}$.

\section{Asynchronous PNC with Linear Cyclic Codes}

In our discussion above, the quasi-cyclic property of convolutional code plays a key role in countering asynchrony. Can other codes that have this property be used to tackle asynchrony? To answer this question, we propose a more general scheme that uses linear cyclic codes to cope with larger-than-one symbol misalignment.

Let $\mathcal{C}(\cdot)$ and $\mathcal{C}^{-1}(\cdot)$ denote the encoding function and decoding function of a particular linear cyclic code (e.g., $\mathrm{BCH}$ code), respectively. Then the encoding process in the end nodes is $C^{i}=\mathcal{C}\left(U^{i}\right), i \in \mathrm{A}, \mathrm{B}$. To ease presentation, we assume BPSK modulation and a symbol misalignment of $\tau$. The received signal at the relay is the overlap of the following two signals:

$$
\begin{aligned}
& \begin{array}{llllll}
x_{1}^{\mathrm{A}} & \cdots & x_{\tau}^{\mathrm{A}} & x_{\tau+1}^{\mathrm{A}} & \cdots & \cdots
\end{array} x_{N}^{\mathrm{A}} \\
& \begin{array}{llllll}
x_{1}^{\mathrm{B}} & x_{2}^{\mathrm{B}} \cdots & x_{N-\tau}^{\mathrm{B}} & x_{N-\tau+1}^{\mathrm{B}} & \cdots & x_{N}^{\mathrm{B}} .
\end{array}
\end{aligned}
$$

Upon receiving the overlapped signal, the relay first aligns the last $\tau$ symbols with the first $\tau$ symbols to obtain a new overlapped signal

$$
\begin{array}{cccccccc}
x_{1}^{\mathrm{A}} & x_{2}^{\mathrm{A}} & \cdots & x_{\tau}^{\mathrm{A}} & x_{\tau+1}^{\mathrm{A}} & \cdots & \cdots & x_{N}^{\mathrm{A}} \\
x_{N-\tau+1}^{\mathrm{B}} & \cdots & \cdots & x_{N}^{\mathrm{B}} & x_{1}^{\mathrm{B}} & x_{2}^{\mathrm{B}} & \cdots & x_{N-\tau}^{\mathrm{B}} .
\end{array}
$$

The result in 260 is actually the signal $X^{\mathrm{A}}+X_{(\tau)}^{\mathrm{B}}$, where $X_{(\tau)}^{\mathrm{B}}$ is the $\tau$-symbol right circular-shifted version of node B's signal. Then the relay can map the signal of 26$)$ to $C^{\mathrm{A}} \oplus C_{(\tau)}^{\mathrm{B}}$, where $C_{(\tau)}^{\mathrm{B}}$ is the $\tau$-bit right circular-shifted version of $C^{\mathrm{B}}$. Note that $C_{(\tau)}^{\mathrm{B}}$ is also a valid codeword due to the property of cyclic code. We assume the source packet corresponding to $C_{(\tau)}^{\mathrm{B}}$ is $\tilde{U}^{\mathrm{B}}$ such that $\tilde{U}^{\mathrm{B}}=\mathcal{C}^{-1}\left(C_{(\tau)}^{\mathrm{B}}\right)$. Because the XOR operator preserves the linearity of codes, the relay first decode the XORed packet by

$$
\begin{aligned}
U^{\mathrm{R}} & =\mathcal{C}^{-1}\left(C^{\mathrm{A}} \oplus C_{(\tau)}^{\mathrm{B}}\right) \\
& =\mathcal{C}^{-1}\left(C^{\mathrm{A}}\right) \oplus \mathcal{C}^{-1}\left(C_{(\tau)}^{\mathrm{B}}\right) \\
& =U^{\mathrm{A}} \oplus \tilde{U}^{\mathrm{B}}
\end{aligned}
$$


and then broadcasts this packet to both the end nodes. After decoding $U^{\mathrm{R}}$ node A first XORs $U^{\mathrm{R}}$ with its own information $U^{\mathrm{A}}$ to obtain $\tilde{U}^{\mathrm{B}}$; then node A re-encodes $\tilde{U}^{\mathrm{B}}$ to obtain $C_{(\tau)}^{\mathrm{B}}=\mathcal{C}\left(\tilde{U}^{\mathrm{B}}\right)$, and left circular-shifts $C_{(\tau)}^{\mathrm{B}}$ to obtain $C^{\mathrm{B}}$; finally from $C^{\mathrm{B}}$ node A can decode $U^{\mathrm{B}}$. For node $\mathrm{B}$, it first right circular-shifts its codeword $C^{\mathrm{B}}$ to produce $C_{(\tau)}^{\mathrm{B}}$ and decodes $C_{(\tau)}^{\mathrm{B}}$ to obtain $\tilde{U}^{\mathrm{B}}$; then it XORs $U^{\mathrm{R}}$ with $\tilde{U}^{\mathrm{B}}$ to obtain $U^{\mathrm{A}}$.

\section{NUMERICAL RESUltS}

We evaluate the performance of the proposed PNC decoding framework under AWGN channel by extensive simulation. First, we compare the BER performances of Jt-CNC, XOR-CD Viterbi, and fullstate Viterbi algorithms in synchronous PNC. Second, we demonstrate the effect of phase offset on our $\mathrm{Jt}-\mathrm{CNC}$ decoder. Third, we show the performance of Jt-CNC algorithm in the presence of symbol and phase asynchrony. Furthermore, we implement the three algorithms in a practical PNC system built on USRP software radio platform, and test them in real indoor environment.

\section{A. BER Performance Comparison}

We compare the BER performances of Jt-CNC, XOR-CD Viterbi (XOR-CDV), and full-state Viterbi (FSV) in synchronous PNC. The XOR-CD Viterbi algorithm and full-state Viterbi algorithm were introduced in Section [II. In the simulations, we adopt convolutional codes of two different code rates: code rate $1 / 2(5,7)$ code and code rate $1 / 3(13,15,17)$ code. We first consider BPSK modulation, assuming AWGN channel.

We plot the BER curve of the full-state Viterbi algorithm as a benchmark for the reduced-state Viterbi algorithm in [13]. In our attempt to replicate the reduced-state Viterbi algorithm, we cannot get the same simulation results in [13] even though we follow the exact specification as described in the paper ${ }^{2}$. Our simulation results are somewhat better than those presented in [13]. To avoid misrepresenting their results, here we just compare the results of full-state Viterbi with Jt-CNC. In [13], a performance gap of $2 \mathrm{~dB}$ was observed between the reduced-state Viterbi and full-state Viterbi. As shown in Fig. 7. Jt-CNC has better BER performance than full-state Viterbi. If the gap between full-state Viterbi and reduced-state Viterbi is $2 \mathrm{~dB}$, then the gap between Jt-CNC and reduced-state Viterbi is at least $2 \mathrm{~dB}$.

\footnotetext{
${ }^{2}$ We believe that there are errors in equation (12) and Fig. 3 in $|13|$. We suspect that in |13|, the SNR was not normalized correctly. Our attempt to contact the authors of [13] by email received no reply.
} 


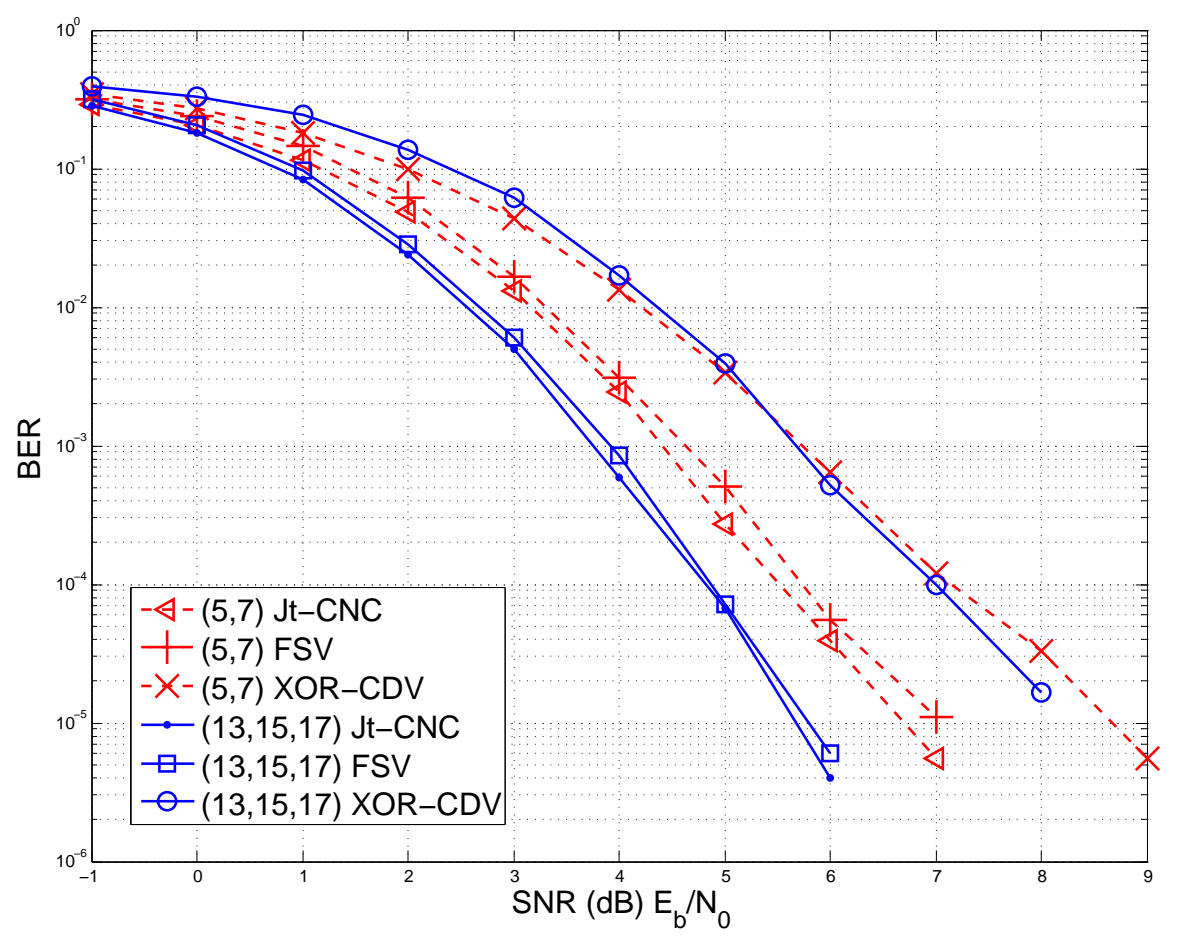

Fig. 7. BER performance of Jt-CNC, XOR-CD Viterbi (XOR-CDV), and full-state Viterbi (FSV) algorithms with PNC. The channel codes are $(5,7)$ and $(13,15,17)$ convolutional code. We use BPSK modulation and assume AWGN channel. 


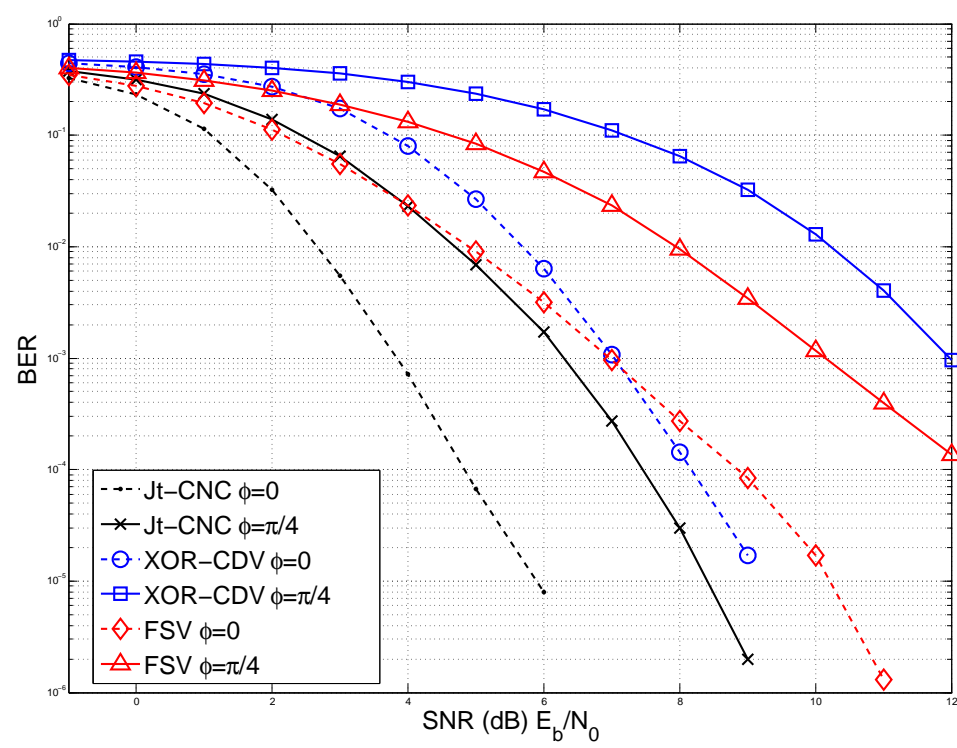

(a) without random-phase precoding

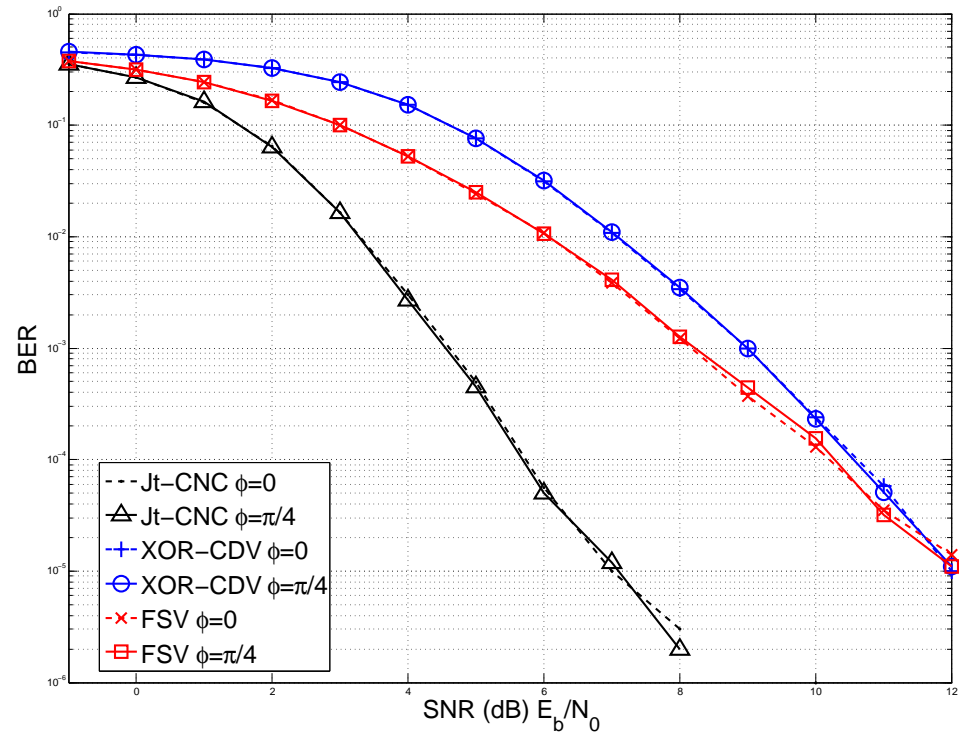

(b) with random-phase precoding

Fig. 8. Effects of phase offset on Jt-CNC, XOR-CD Viterbi (XOR-CDV), and full-state Viterbi (FSV). QPSK modulation and $(13,15,17)$ convolutional code are used in the simulation. We assume the symbols are aligned and the relative phase offset is $\pi / 4$. In (a), both nodes transmit their signals directly; in (b), node B precodes its transmit signal with a pseudo-random phase sequence.

As shown in Fig. 8, phase offset degrades the performances of all the three algorithms. Jt-CNC is more resistant to phase penalty because the joint processing makes better use of the soft information. We note the performance of Jt-CNC under the worst phase offset of $\pi / 4$ is still better than the performance of XOR-CDV with no phase offset.

To improve our system's resilience against phase offset, we adopt the random-phase precoding at the transmitter of one end node. Specifically, node B rotates the phase of its transmitted signal with a pseudorandom phase sequence $\Phi^{\mathrm{B}}=\left(\phi_{1}^{\mathrm{B}}, \cdots, \phi_{N}^{\mathrm{B}}\right)$ where $\phi_{n}^{\mathrm{B}}$ is randomly chosen from zero to $\pi / 4$. We assume that this pseudo-random phase sequence is known at the relay so that it can incorporate this knowledge into the decoding process. As shown in Fig. $8 \mathrm{~b}$ with the random-phase precoding algorithm, the phase penalty is reduced to $1 \mathrm{~dB}, 1 \mathrm{~dB}$, and $3 \mathrm{~dB}$ for Jt-CNC, FSV, and XOR-CDV, respectively.

\section{Effects of Symbol Misalignment}

A major advantage of the proposed decoding framework is that it can deal with general symbol misalignment. We evaluate the performance of Jt-CNC under varying degrees of symbol misalignment 
and phase offset. In the simulation, both end nodes transmit 1000-bit source packets (corresponding to 1500 QPSK symbols for channel code rate of $1 / 3$ ).

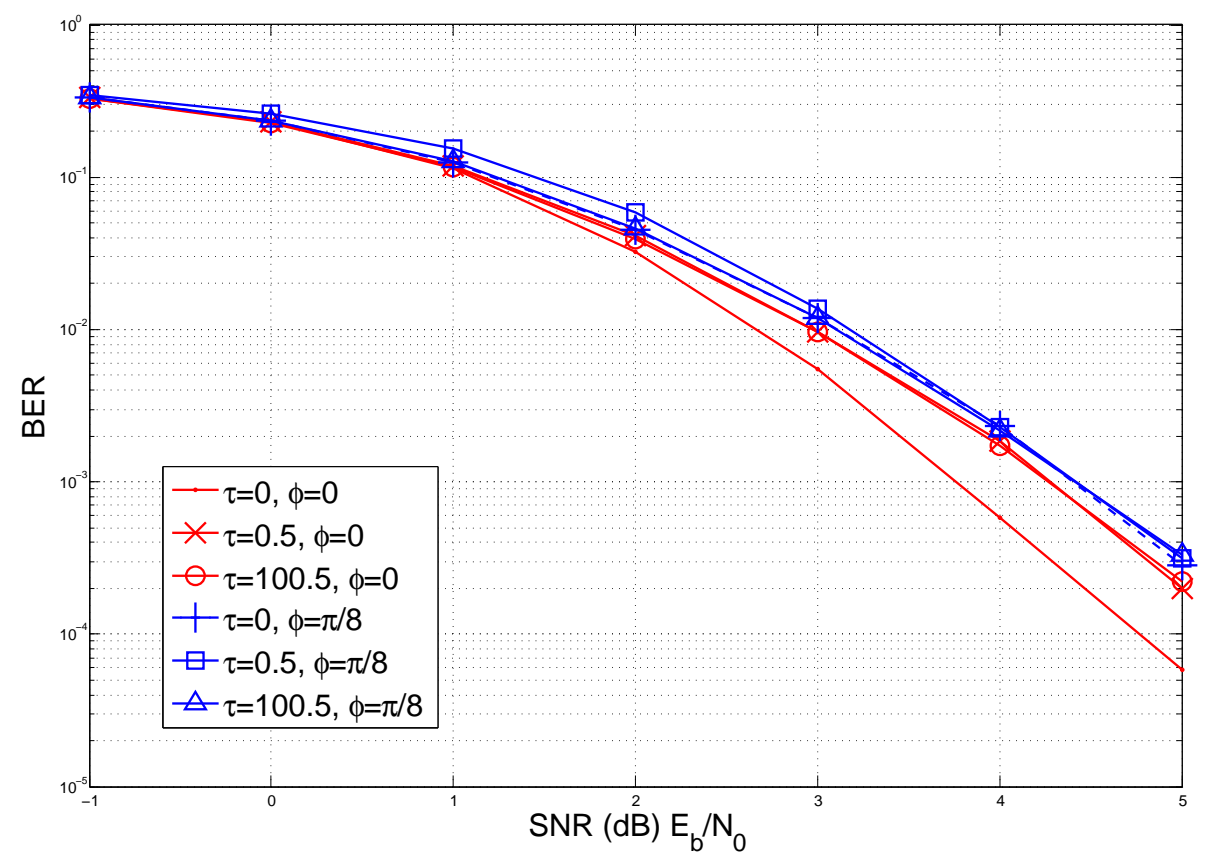

Fig. 9. BER performance of Jt-CNC decoder under general symbol misalignment, with $(13,15,17)$ convolutional code and QPSK modulation.

\section{Software Radio Experiment}

To evaluate the proposed algorithm in a real communication system, we implemented a PNC system using USRP N210, embedded with the three decoding algorithms. The PNC system adopts OFDM modulation with $1 \mathrm{MHz}$ bandwidth and $2.48 \mathrm{GHz}$ carrier frequency. We use the $(5,7)$ convolutional code and follow the frame format design in [8]. We conduct our experiments in the indoor office environment and evaluated the BER performance of Jt-CNC, XOR-CD, and full-state Viterbi algorithms under different SNRs. In the experiment, we balanced the powers of the end nodes and let both nodes transmit 500 frames 

data).

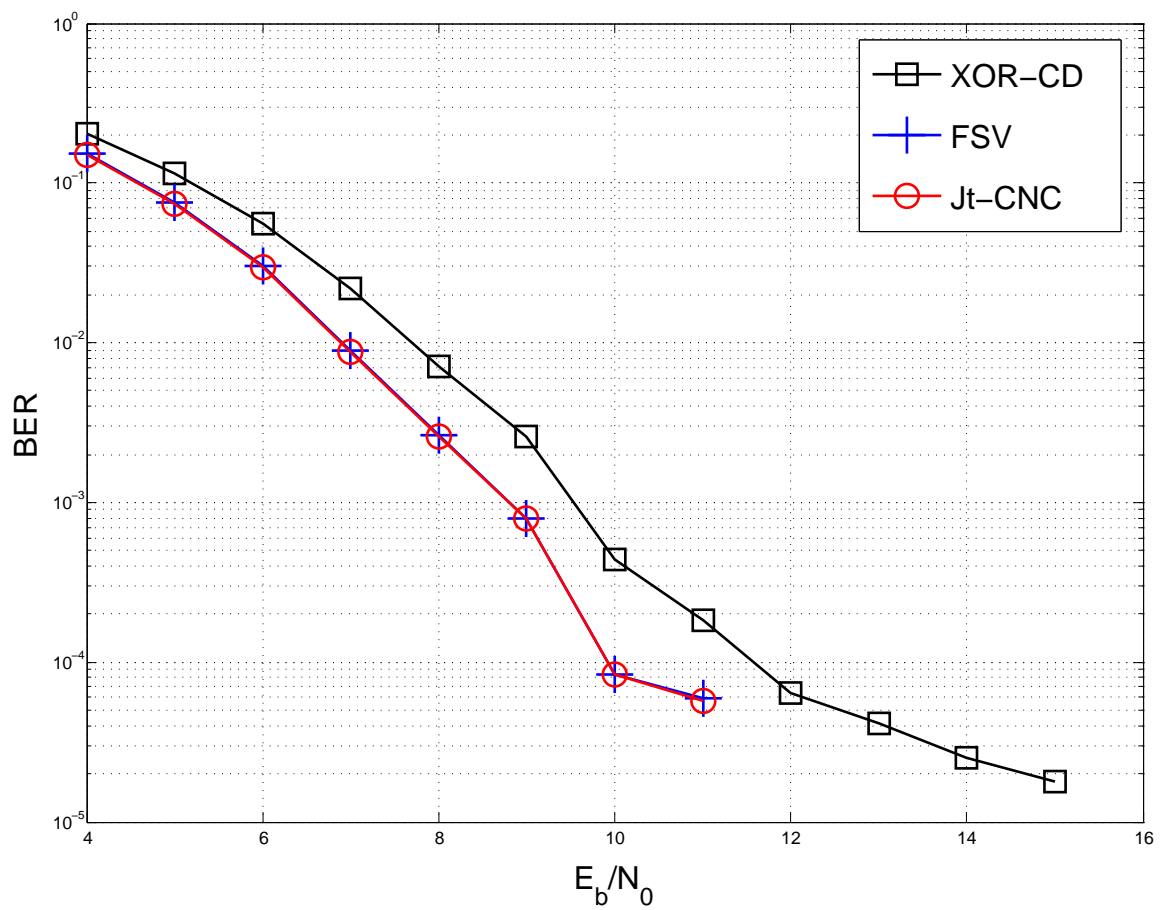

Fig. 10. BER performance of Jt-CNC, FSV, and XOR-CD in an indoor environment. We tested the three algorithms on a practical PNC system implemented on USRP N210. The PNC system adopts OFDM modulation and (5, 7) convolutional code.

We have proposed a three-layer decoding framework for asynchronous convolutional-coded PNC systems. This framework can deal with general (integral plus fractional) symbol misalignment in convolutionalcoded PNC systems. Furthermore, we design a Jt-CNC algorithm to achieve the BER-optimal decoding of convolutional code in synchronous PNC. For asynchronous PNC, the performance degradation is 

1 convolutional code is

$$
\mathbf{G}=\left[\begin{array}{cccccccc}
\mathbf{g}_{0} & \mathbf{g}_{1} & \mathbf{g}_{2} & \cdots & \mathbf{g}_{m} & & & \\
& \mathbf{g}_{0} & \mathbf{g}_{1} & \cdots & \mathbf{g}_{m-1} & \mathbf{g}_{m} & & \\
& & \ddots & & & & \ddots & \\
& & & \mathbf{g}_{0} & \mathbf{g}_{1} & \mathbf{g}_{2} & \cdots & \mathbf{g}_{m} \\
\mathbf{g}_{m} & & & & \mathbf{g}_{0} & \mathbf{g}_{1} & \cdots & \mathbf{g}_{m-1} \\
\mathbf{g}_{m-1} & \mathbf{g}_{m} & & & & \mathbf{g}_{0} & \cdots & \mathbf{g}_{m-2} \\
\vdots & & \ddots & & & & \ddots & \\
\mathbf{g}_{1} & \mathbf{g}_{2} & \cdots & \mathbf{g}_{m} & & & & \mathbf{g}_{0}
\end{array}\right]
$$


2

where $\mathbf{g}_{\mathrm{b}}=\left[\begin{array}{llll}\mathbf{g}_{0} & \mathbf{g}_{1} & \cdots & \mathbf{g}_{m}\end{array}\right]$ is the basis generator matrix of the convolutional code; each entry $\mathbf{g}_{i}$ is an $R$-bit vector

$$
\mathbf{g}_{i}=\left[\begin{array}{llll}
g_{i}^{(1)} & g_{i}^{(2)} & \cdots & g_{i}^{(R)}
\end{array}\right]
$$

where $g_{i}^{(r)}$ is equal to 1 or 0 , corresponding to whether the $i$ th stage of the shift register contributes (connects) to the $r$ th output. Therefore, the basis generator matrix $\mathbf{g}_{\mathrm{b}}$ can be regarded as the "impulse response" of the convolutional encoder. For example, the basis generator matrix of $(5,7)$ convolutional code shown in Fig. 11 is $\left[\begin{array}{lll}11 & 01 & 11\end{array}\right]$. The encoding process is simply

$$
C=U \mathbf{G}
$$

The right circular-shifted codeword can be represented by

$$
C_{(k R)}=U \mathbf{G}_{(k)}
$$

where $\mathbf{G}_{(k)}$ is obtained by right circular-shift matrix $\mathbf{G}$ by $k \times R$ columns. Since $\mathbf{G}$ is a circulant matrix, we have

$$
C_{(k R)}=U \mathbf{G}_{(k)}=U_{(k)} \mathbf{G} .
$$

Therefore the source packet of $C_{(k R)}$ is $U_{(k)}$, the $k$-bit circular-shifted version of $U$.

Remark 1: Theorem 1 is also valid for the tail biting convolutional code with a general code rate $L / R L, R \in \mathbf{N}^{+}$, but the resulting source packet will be $U_{(k L)}$, the $k L$-bit right circular-shifted version of $U$. The proof is the same except that the entry of the basis generator matrix $\mathbf{g}_{\mathbf{b}}$ is an $L \times R$ matrix:

$$
\mathbf{g}_{i}=\left[\begin{array}{cccc}
g_{1, i}^{(1)} & g_{1, i}^{(2)} & \cdots & g_{1, i}^{(R)} \\
g_{2, i}^{(1)} & g_{2, i}^{(2)} & \cdots & g_{2, i}^{(R)} \\
\vdots & \vdots & & \vdots \\
g_{L, i}^{(1)} & g_{L, i}^{(2)} & \cdots & g_{L, i}^{(R)}
\end{array}\right]
$$

where $g_{l, i}^{(r)}$ is equal to 1 or 0 , depending on whether the $i$ th stage of the shift register for the $l$ th input contributes (connects) to the $r$ th output.

Theorem 2: If the initial state and terminal state of a convolutional code are the same, then the decoding output of $C_{(k R)}$, the $k R$-bit right circular-shifted version of $C$, is the $k$-bit right circular-shifted version of $U$.

Proof: The encoding and decoding process of a convolutional code can be represented by the Tanner graph in Fig. 2. Since the code has the same initial and terminal state, we can merge the Tanner graph 


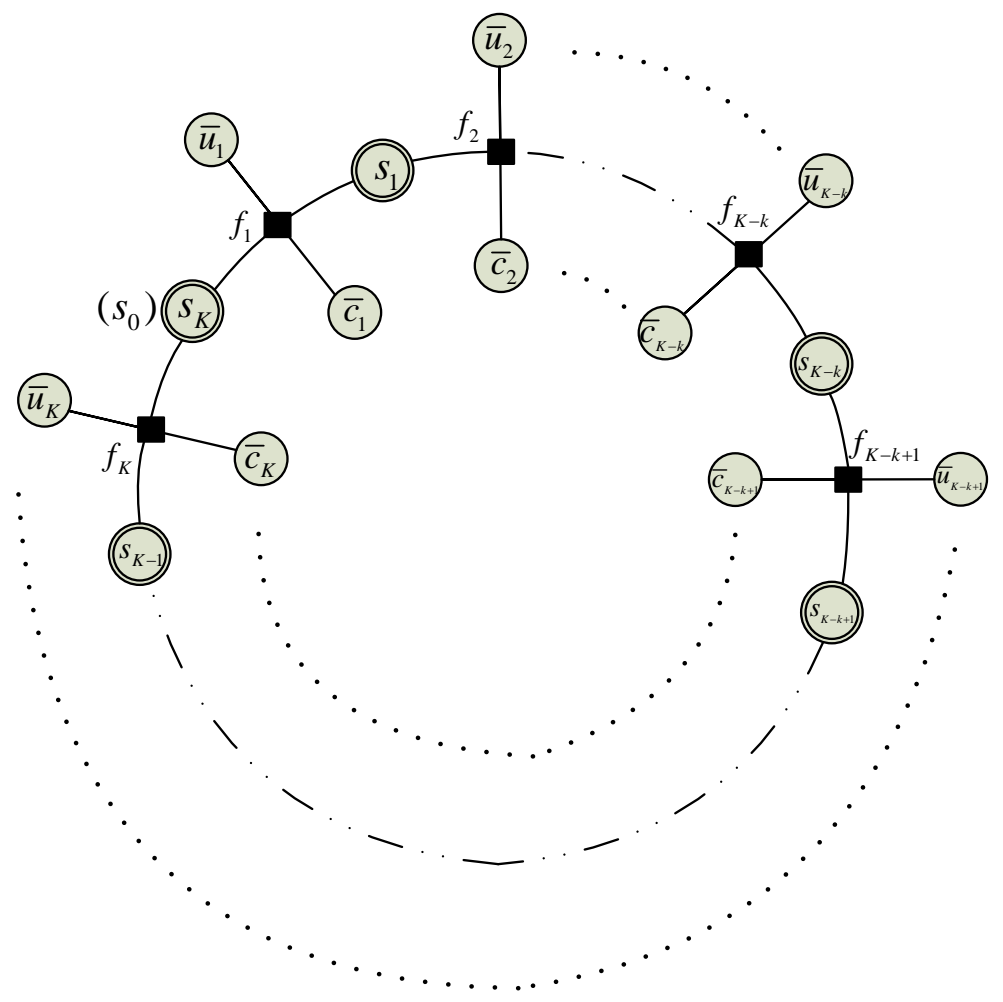

Fig. 12. Tanner graph of a convolutional code that has the same initial state $S_{0}$ and terminal state $S_{K}$. We merge the initial state and terminal state, hence the Tanner graph in Fig. 2 becomes a ring.

as shown in Fig. 12, For a general convolutional code with code rate $L / R$, the source message $\bar{u}_{k}$ is an $L$-bit tuple and the coded message $\bar{c}_{k}$ is an $R$-bit tuple.

Let $C_{(k R)}=\left(\bar{c}_{K-k+1}, \bar{c}_{K-k+2}, \cdots, \bar{c}_{K}, \bar{c}_{1}, \cdots, \bar{c}_{K-k}\right)$ be the $k R$-bit right circular-shifted version of codeword $C$. To decode $C_{(k R)}$, the decoding algorithm starts with the first tuple $\bar{c}_{K-k+1}$ and ends with the last tuple $\bar{c}_{K-k}$. Because the Tanner graph has a ring structure, the decode output is $U_{(k)}=$ $\left(\bar{u}_{K-k+1}, \bar{u}_{K-k+2}, \cdots, \bar{u}_{K}, \bar{u}_{1}, \cdots, \bar{u}_{K-k}\right)$, which is the $k$-bit right circular-shifted version of $U$.

Remark 2: Zero tailing convolutional codes also have the property in Theorem 2, because zero tailing convolutional code has zero initial state and zero terminal state.

Remark 3: For a recursive convolutional code, we can append zero-tailing bits to the input packet to make the terminal state zero state. Then recursive convolutional codes can also be used with the proposed Jt-CNC decoder.

\section{ACKNOWLEDGMENT}

The authors would like to thank... 


\section{REFERENCES}

[1] S. Zhang, S. C. Liew, and P. P. Lam, "Hot topic: physical-layer network coding," in Proceedings of Mobicom 2006. ACM, 2006, pp. 358-365.

[2] R. Ahlswede, N. Cai, S.-Y. Li, and R. W. Yeung, "Network information flow," IEEE Trans. Inf. Theory, vol. 46, no. 4, pp. 1204-1216, 2000.

[3] S.-Y. Li, R. W. Yeung, and N. Cai, "Linear network coding," IEEE Trans. Inf. Theory, vol. 49, no. 2, pp. 371-381, 2003.

[4] S. Zhang and S. C. Liew, "Channel coding and decoding in a relay system operated with physical-layer network coding," IEEE J. Sel. Areas Commun., vol. 27, no. 5, pp. 788-796, 2009.

[5] L. Lu and S. C. Liew, "Asynchronous physical-layer network coding," IEEE Trans. Wireless Commun., vol. 11, no. 2, pp. 819-831, 2012.

[6] IEEE-SA Standards Board, "Wireless LAN medium access control (MAC) and physical layer (PHY) specifications," IEEE Std 802.11 part 11, 2003.

[7] L. Lu, T. Wang, S. C. Liew, and S. Zhang, "Implementation of physical-layer network coding," Physical Communication, 2012.

[8] L. Lu, L. You, Q. Yang, T. Wang, M. Zhang, S. Zhang, and S. C. Liew, "Real-time implementation of physical-layer network coding," in Proceedings of the 2nd Workshop on Software Radio Implementation Forum. ACM, 2013, pp. 71-76.

[9] F. Rossetto and M. Zorzi, "On the design of practical asynchronous physical layer network coding," in IEEE 10th Workshop on SPAWC '09. IEEE, 2009, pp. 469-473.

[10] S. C. Liew, S. Zhang, and L. Lu, "Physical-layer network coding: Tutorial, survey, and beyond," Physical Communication, vol. 6, pp. 4-42, 2013.

[11] A. Viterbi, "Error bounds for convolutional codes and an asymptotically optimum decoding algorithm," IEEE Trans. Inf. Theory, vol. 13, no. 2, pp. 260-269, 1967.

[12] L. Bahl, J. Cocke, F. Jelinek, and J. Raviv, “Optimal decoding of linear codes for minimizing symbol error rate,” IEEE Trans. Inf. Theory, vol. 20, no. 2, pp. 284-287, 1974.

[13] D. To and J. Choi, "Convolutional codes in two-way relay networks with physical-layer network coding," IEEE Trans. Wireless Commun., vol. 9, no. 9, pp. 2724-2729, 2010.

[14] D. Wang, S. Fu, and K. Lu, "Channel coding design to support asynchronous physical layer network coding," in Proceedings of Globecom 2009. IEEE, 2009, pp. 1-6.

[15] X. Wu, C. Zhao, and X. You, "Joint ldpc and physical-layer network coding for asynchronous bi-directional relaying," IEEE J. Sel. Areas Commun., vol. 31, no. 8, pp. 1446-1454, 2013.

[16] H. Ma and J. Wolf, "On tail biting convolutional codes," IEEE Trans. Commun., vol. 34, no. 2, pp. 104-111, 1986.

[17] V. Namboodiri, K. Venugopal, and B. S. Rajan, "Physical layer network coding for two-way relaying with QAM," arXiv preprint arXiv:1301.4646, vol. abs/1301.4646, 2013. [Online]. Available: http://arxiv.org/abs/1301.4646

[18] H. J. Yang, Y. Choi, and J. Chun, "Modified high-order PAMs for binary coded physical-layer network coding," IEEE Commun. Lett., vol. 14, no. 8, pp. 689-691, 2010.

[19] T. Koike-Akino, P. Popovski, and V. Tarokh, "Optimized constellations for two-way wireless relaying with physical network coding," IEEE J. Sel. Areas Commun., vol. 27, no. 5, pp. 773-787, 2009. 
2 [20] J. Pearl, Probabilistic reasoning in intelligent systems: networks of plausible inference. Morgan Kaufmann, 1988.

3 [21] F. R. Kschischang, B. J. Frey, and H.-A. Loeliger, "Factor graphs and the sum-product algorithm," IEEE Trans. Inf. Theory, vol. 47, no. 2, pp. 498-519, 2001.

[22] M. Esmaeili, T. A. Gulliver, N. P. Secord, and S. A. Mahmoud, "A link between quasi-cyclic codes and convolutional codes," IEEE Trans. Inf. Theory, vol. 44, no. 1, pp. 431-435, 1998.

[23] G. Solomon and H. Tilborg, "A connection between block and convolutional codes," Journal on Applied Mathematics, vol. 37, no. 2, pp. 358-369, 1979.

9

\begin{tabular}{|c|}
\hline \\
PLACE \\
PHOTO \\
HERE \\
\hline
\end{tabular}

xxxx Biography text here.

$\mathbf{x x x x}$ Biography text here.

$\mathbf{x x x x}$ Biography text here.
DRAFT 